\title{
LE TRAVAIL DU RÉCIT SUR LES RELATIONS DE CARE: LITTÉRATURE ET SOCIOLOGIE
}

\section{Writing stories about care work: literature and sociology}

\author{
Laura Marzi*; Patricia Paperman** \\ * CNRS / UPL (Paris 8 Vincennes Saint-Denis / Paris Ouest Nanterre La Défense); ** Université Paris 8, UMR \\ LEGS 8238; CNRS / UPL (Paris 8 Vincennes Saint-Denis / Paris Ouest Nanterre La Défense) (Francia)
}

lallacoto@gmail.com; patriciapaperman@wanadoo.fr

Keywords

Care

Narration

Heroine

Ethnography

\begin{abstract}
From a feminist care perspective, this article analyses how literary and ethnographic approaches represent care work and the relationship between the domestic employee and the employer, thus contributing to make visible the subaltern position of the careworker. The work of literature is illustrated through the example of the novel The Door by Magda Szabò. In this novel the starting point is the will of the writer to repair her betrayal towards the domestic worker Emerenc. Writing a novel, telling Emerenc 's life story, thus making her visible, is the way for Szabò to accomplish this reparation. Does this reparation comes from heroisation? In care novels, care-givers occupy the main stage: they are heroines, not in the sense of universal male heroism, but in one that emerges from the care stories read from a gender perspective. The ethnographic approach is considered through the work of Caroline Ibos Qui gardera nos enfants? This fieldwork is an inquiry about the relationship between Ivorian nannies and woman employers in the Paris region. The book relies mainly on the way the nannies tell their lives and migration. It shows why and how the employers need to keep invisible the daily presence of the foreign care worker in order to maintain intact the symbolic order of their appartement.
\end{abstract}

\begin{abstract}
Mot clés
Care

Récit

Héroïne

Ethnographie

Résumé

A partir de la perspective féministe du care, cet article propose une analyse mettant en regard la façon dont les approches littéraire et ethnographique parviennent à représenter la position subalterne de la travailleuse du care et la relation de care entre l'employée et l'employeuse domestique. Le travail de la littérature est étudié à partir du roman La Porte de Magda Szabò. Dans cet ouvrage, le déclenchement de l'histoire est la volonté de la part de l'écrivaine de réparer sa trahison envers l'employée domestique Emerenc. Cette réparation consiste pour Szabò dans l'écriture du récit de l'histoire de la vie d'Emerenc, qui devient ainsi visible. On se demande ensuite si cette réparation passe à travers une héroïsation du personnage de la travailleuse. Dans les romans de care les femmes care-givers occupent la scène principale: elles sont des héroïnes, non pas dans le sens de l'héroïsme universel masculin, mais de celui qui émerge des récits de care au prisme du genre. L'approche ethnographique est considérée à partir du livre de Caroline Ibos Qui gardera nos enfants? Cette enquête de terrain sur les relations entre nounous ivoiriennes et employeuses, en région parisienne laisse une large place aux paroles des nounous. Elle montre comment l'invisibilisation de la travailleuse du care répond à la nécessité pour l'employeuse de garder intact l'ordre symbolique de son appartement en dépit du passage quotidien de la femme étrangère.
\end{abstract}

Marzi, L., Paperman, P., 2016, "Le travail du récit sur les relations de care: littérature et sociologie", en Papeles del CEIC, vol. 2016/1, no 149, CEIC (Centro de Estudios sobre la Identidad Colectiva), Universidad del País Vasco, http://dx.doi.org/10.1387/pceic.15425 
Les travaux qui, dans le monde occidental, développent une perspective féministe à partir du concept de care se sont concentrés de façon massive sur l'analyse du travail de care assumé par les catégories sociales subalternes. L'invisibilité de ce travail, de son importance pour le maintien de la vie humaine dans des conditions diverses d'organisation sociale a été la cible de ces analyses. Un des effets et un des objectifs de ces études a été de rendre visible un travail invisible. Mais qu'est ce qui, de ce travail, est rendu visible par les études en sciences sociales?

L'importance des données chiffrées sont décisives dans les argumentations et le débat public quand il s'agit de (dé)montrer la réalité d'un phénomène social et l'acuité d'un problème social. Les sociologues du travail du care ont utilisé dans ce sens l'outil des chiffres, tout en les inscrivant dans des analyses appuyées sur des différentes théorisations, celles des transformations du travail, des migrations transnationales, de la division internationale du travail et du genre ${ }^{1}$.

À la différence de l'approche sociologique dans ses versions structurales et macrologiques, l'approche ethnographique comme méthode pour la sociologie fournit des récits centrés sur des situations concrètes et des personnes singulières, sur des relations sociales. En ce sens, approche ethnographique et approche littéraire peuvent être envisagées ensemble en tant qu'elles racontent des histoires ou mettent des vies des morceaux de vie- en récit. Les considérer ensemble, dans leur proximité et leur spécificité, permet peut-être - telle est l'ambition de notre texte- de comprendre mieux, en tous cas autrement, ce que veulent dire ces expressions si fréquemment lues et utilisées, le plus souvent de façon allusive ou métaphorique: "invisibiliser" ou à l'inverse "rendre visible le travail du care".

Nous présentons ici le fruit d'une conversation à deux voix sur la façon dont récits littéraires et comptes rendus ethnographiques sur le travail de care sollicitent l'attention des lectrices et lecteurs sur les travailleuses du care et sur leur position d'invisibilité. Si les deux approches, littéraire et ethnographique, peuvent être envisagées ensemble parce qu'elles visent à faire apparaître les travailleuses du care dans leurs relations

\footnotetext{
${ }^{1}$ Cf.: Hirata, H., avec Labari, B., Falquet, J., Le Feuvre, N., Kergoat, D., Sow, F., (Eds), 2010, Le sexe de la mondialisation. Genre, classe, race et nouvelle division du travail, Presses de Sciences Po, coll. Académique, Paris.
} 
avec les employeuses, elles emploient des registres différents de description. Et, ainsi, elles donnent à "voir" des aspects différents de l'invisibilisation et de la visibilisation des positions des travailleuses domestiques.

Nous avons choisi le roman La Porte de Magda Szabò parce qu'il raconte la relation de care entre une femme de ménage et une écrivaine, qui la transforme dans l'héroïne de son texte. L'ouvrage ethnographique Qui gardera nos enfants? de Caroline Ibos problématise, du point de vue politique, justement cette héroïsation.

\section{CE Que FAIT LA LITTÉRATURE}

Le récit littéraire de la relation qui se développe entre les deux femmes, une employeuse et une employée domestique, apporte une vision sur les expériences du travail que l'une fait pour une autre qui la paye, il donne également une vision de la dimension affective et éthique qui compose la relation inégale entre employeuse et employée. Et surtout il rend visible ce qui précisément ne l'est pas dans la relation inégale: la personne, son histoire, sa vie avant et en dehors de la relation à l'employeuse. Dans le roman La porte de l'écrivaine hongroise Magda Szabò (1987) I'histoire d'Emerenc, l'employée domestique de Magda (dans le roman le prénom de la narratrice n'apparait jamais, mais nous pouvons déduire des épisodes racontés qu'il s'agit de la vie de l'auteure Magda Szabò) est un des enjeux de la relation entre les deux femmes. Emerenc travaille pour Magda et pour des autres employeuses dans le quartier: "cette vieille femme travaillait comme un robot, elle soulevait sans se ménager des meubles apparemment inamovibles, sa force de travail et son énergie avaient quelque chose de surhumain" (Szabò, 2003: 16). Son histoire est cachée tout au long du roman par Emerenc derrière la porte de sa maison qu'elle n'ouvre à personne et qu'elle finira par ouvrir à Magda: "je vous ai laissé entrer là où personne n'est jamais entré. Je ne peux rien vous offrir de plus, parce que je n'ai rien d'autre" dit Emerenc (Szabò, 2003:178).

Emerenc, qui détient les clés des maisons de plusieurs personnes chez lesquelles elle fait le ménage, empêche tout le monde d'entrer chez elle. Peut-être agit-elle ainsi aussi parce qu'à cause de son travail elle est consciente des conséquences que comporte le fait de laisser quelqu'une entrer dans la maison. En franchissant la porte des maisons des autres, 
elle entre en contact avec leur intimité. Lorsqu'elle fait le ménage chez les autres, elle touche avec ses mains, par exemple, les linges où les employeuses-eurs dorment et où, dans certains cas, ils-elles sont nu-e-s et font l'amour; ou bien où ils-elles perdent le contrôle de leurs corps, et, comme on le dit, leur dignité (expression injuste, pour décrire les conséquences de la perte du contrôle par rapport à nos besoins physiologiques). Ses mains lavent la baignoire, le bac de la douche, la cuvette des toilettes, et touchent là encore les espaces domestiques utilisés par les employeuses et les employeurs nu-e-s.

Cependant, Emerenc permet à Magda de franchir la porte de chez elle. Pour les lectrices et les lecteurs le moment d'ouverture de la porte, ce dernier dévoilement, constitue l'aboutissement d'un processus de connaissance de l'histoire d'Emerenc que la vieille femme a livrée à Magda petit à petit, tout au long de leur relation. Emerenc, avant l'ouverture de la porte, lui a raconté l'histoire tragique de son enfance, des épisodes de sa vie pendant la guerre, ses amours et ses désillusions. Ainsi, le mûrissement de l'amour réciproque entre les deux femmes, déclaré par Emerenc quelques pages avant l'ouverture de la porte, coïncide avec l'accomplissement du don de son histoire à Magda.

On pourrait lire dans l'incapacité de Magda de savoir mériter ce don, le point de départ du roman, ce qui pousse Szabò à nous raconter cette histoire. Dans les premières pages du livre l'auteure exprime la nécessité d'écrire cette histoire pour donner voix à l'angoisse de n'avoir pas su endosser la responsabilité de l'énorme confiance que la vieille femme lui avait faite, de n'avoir pas su répondre à un amour si fort: "celle qui tournait la clé croyait davantage en moi, qu'en Dieu, et moi, en cet instant fatal, je croyais être Dieu, sage, pondérée, bonne et rationnelle. Nous étions toutes deux dans l'erreur, elle, qui avait confiance en moi, et moi qui pêchais par excès d'assurance" (Szabò, 1993: 8).

Emerenc a une crise d'apoplexie à la suite de laquelle elle reste renfermée chez elle, ne permettant à personne d'entrer. Cet épisode coïncide avec un moment crucial de la carrière de Magda, qui, après une longue période pendant laquelle son pays avait méprisé son œuvre, reçoit un prix littéraire très prestigieux. C'est pour cette raison qu'elle ne prête pas suffisamment attention au comportement d'Emerenc, déjà malade, qui refusait de lui ouvrir la porte ou de sortir pour lui parler et surtout, qui avait cessé de travailler. Mais en réalité Emerenc ne sort pas de chez elle et ne travaille plus parce qu'elle est paralysée comme on le 
découvrira quand, à la suite d'une ruse, les gens du quartier, grâce à l'aide de Magda, réussissent à la faire sortir de son appartement et à l'amener à l'hôpital. Mais sa maison est profanée, son trésor (ses chats et ses meubles) est perdu et la vieille femme ne supporte pas la douleur: elle meurt à l'hôpital. N'ayant pas su honorer ce geste d'amour, Magda décide de réparer en racontant l'histoire d'Emerenc. Mais comment le récit peut-il constituer une réparation?

Comme le dit Teresa de Lauretis "what is finally at stake is not how much to make visible the invisible as how to produce the conditions of visibility for a different social subject" (1982: 9). Ainsi, Magda comprend que pour réparer son tort, son incapacité de sauvegarder le secret dont Emerenc lui avait fait don comme la chose la plus précieuse qu'elle possédait, elle ne peut qu'ouvrir la porte et rendre visible Emerenc pour tout le monde. Ce roman naît de la volonté de Magda de témoigner de la centralité du rôle d'Emerenc dans sa vie. En la transformant en héroïne de son histoire, Magda rend Emerenc importante, pour nous également, ses lectrices et lecteurs. En même temps, elle montre l'héroïsme ordinaire d'une employée domestique qui franchit les portes des autres pour y affronter maladie, handicap, saleté, vieillesse, dépendance. La réparation passerait-elle par l'héroïsation?

Avec son roman Magda Szabò transforme en héroïne, une femme, une bonne à tout faire, une employée domestique qui est peut-être considérée comme une personne formidable par son entourage, mais qui est inconnue du reste du monde. Mais qu'est-ce qu'une héroïne?

La catégorie d'héroïne est une catégorie critique façonnée sur celle de héros: "la culture héroïque se présente comme une célébration de la masculinité. Non seulement les femmes y tiennent une moindre place, mais on peut dire de cette culture qu'elle se construit à partir de la répression du pôle féminin, ou du moins, de sa subordination" (Apostolidès, 2011: 46). Le terme héroïne, en fait, n'est pas l'équivalent féminin de celui de héros, duquel dérive le concept d'héroïsation qui, d'après le Dictionnaire Culturel en langue française, est l'"action d'héroïser". Héroïser est défini ainsi: "1. Élever au rang de héros. 2. Attribuer le caractère d'un héros" (2005: 1611). Par ailleurs, I'héroïsation est définie par Le Grand Larousse de la langue française comme I'"action d'héroïser quelqu'un: apothéose d'un héros" (1973: 2414), tout comme Le Grand Larousse Encyclopédique (1962: 869). 
Le personnage de la care-giver, protagoniste de récits littéraires de care, est une héroïne parce qu'elle est la protagoniste de l'histoire, mais aussi parce que, par ces actions, elle contribue à la survie des care-receivers. Elle ne le fait pas, comme c'est le cas des super héros, en tuant les méchants ou en libérant la Terre du Mal, mais en s'occupant des besoins quotidiens de personnes qui seules n'y arrivent pas. L'héroïne Emerenc garantit la survie de Magda et de son mari, par exemple.

Dans un autre roman de care, Marijana, protagoniste de Slow Man de J.M. Coetzee (2005), s'occupera de Paul, l'homme unijambiste et de sa maison... Cependant, l'héroïsme est une catégorie neutre-masculine qui empêche d'analyser le personnage de la care-giver en tant que héroïne, en premier lieu parce qu'elle est une femme.

Les critiques littéraires Loïse Bilat et Gianni Haver dans le texte Le héros était une femme décident de ne pas utiliser le terme "héroïne", mais de se servir de la périphrase "héros féminin":
"Le terme héroïne nous semble peu satisfaisant pour caractériser un personnage féminin qui liquide une centaine de combattant-e-s des triades japonais, à coup de sabre -ils se réfèrent à Béatrix Kiddo, le personnage interprété par Uma Thurman dans le film Kill Bill Volume 1 et à son exploit de violence qui lui permet de tuer les 88 criminels de la Yakuza, aux ordres de O-Ren Ishii-. Nous préférons le syntagme de héros féminin pour parler de nos personnages, faute de mieux. Nous pouvons conclure, en effet, que la féminisation ne suffit pas à établir une égalité sémantique entre héros et héroïne, comme c'est le cas pour d'autres signifiants" (Bilat et Haver, 2011: 18).

Le Trésor de la langue Française pour l'article "héroïne" renvoie à "héros", la définition du mot "héroïne" se référant à la drogue. Pour l'article "héros" il y a deux acceptions: une valable seulement pour "héros": "1. Nom donné par les Grecs aux demi-dieux ou aux grands hommes divinisés; 2. Personnage légendaire à qui l'on prête des exploits extraordinaires"; l'autre valable aussi pour "héroïne": "personne qui se distingue par des qualités ou des actions exceptionnelles, par son courage face au danger: mourir en héros. 2. Personnage principal d'une œuvre de fiction, d'un événement: elle a été l'héroïne involontaire d'un fait divers" (1981: 3421).

Dans Le Grand Larousse Encyclopédique, on trouve une brève définition de "héroïne": "sert de féminin à héros (...) Personnage principal du sexe 
féminin dans une action réelle ou fictive". Ensuite on a cent-vingt-cina lignes dédiées à la définition de héros dans la mythologie; dans l'iconographie et par rapport aux catégories: héros de notre temps; héros du travail; héros du travail socialiste; héros de l'union soviétique (1962: 869).

Dans Le Nouveau Petit Robert on trouve un article spécifique pour héroïne "femme d'un grand courage, qui fait preuve par sa conduite, dans des circonstances exceptionnelles, d'une force d'âme au-dessus du commun, voir héros. 2. Principal personnage féminin d'une œuvre" (2008: 924-925). On a vu comment le Trésor de la Langue Française spécifie le caractère involontaire de l'héroïsme d'une héroïne, ici on peut noter que selon le Petit Robert une héroïne "fait preuve de courage" mais seulement "dans des circonstances exceptionnelles". Mais Le Nouveau Petit Robert, à la différence du Grand Larousse et du Trésor de la Langue Française, propose une notice spécifique pour le mot héroïne. Cependant les trois dictionnaires, quand ils citent le courage héroïque, donnent des exemples seulement au masculin "glorieux, vaillant héros; héros de la Grande Armée, de la Révolution (...) mourir, se comporter en héros" (Le Trésor de la langue française, 1981: 797); "les héros de la Grande Guerre" (Grand Larousse de la langue française, 1989: 2414); "combattants qui meurent, qui tombent en héros" (Le Nouveau Petit Robert, 2008: 1231).

Enfin, les trois dictionnaires attribuent tous à "héroïne" le sens de personnage principal d'une scène ou d'une fiction, ils explicitent que cette définition se réfère autant à héros qu'à héroïne.

Du point de vue de la critique littéraire, la difficulté évoquée par Loïse Bilat et Gianni Haver du manque d'égalité sémantique entre les termes "héros" et "héroïne" que nous avons noté précédemment, est soulignée également par Deborah Lions:

"The word heroine carries out with an unfortunate freight of associations, suggesting not a powerful being to be invoked and propitiated from beyond the grave, but a frail creature requiring rescue by none other than a hero. I decided not to circumvent this problem by the use of the phrase 'female hero', since such a phrase renforces the notion of the female as the special case, the other, the marked category, while the male remains unmarked, normative, universal" (Lions, 1987: 4). 
Comme l'écrit cette critique littéraire anglo-saxonne, si on ne s'interroge pas sur ce qu'est une héroïne et quel est l'héroïsme qui la caractérise, on se condamne à accepter que le concept proposé d'héroïsme est universel, quand il est, en vérité, masculin. En effet, par rapport à la définition du mot héroïsme les dictionnaires de la langue françaises que j'ai consultés sont tous cohérents: ils renvoient au mot "héros", ils ne s'expriment donc pas sur l'héroïsme des femmes.

Les héroïnes des histoires de care, les protagonistes travailleuses domestiques, peuvent représenter un véritable défi par rapport à I'hérö̈sme neutre masculin et à son élan vers la mort. En effet, leurs actions, donc leur héroïsme, sont accomplies pour la sauvegarde de la vie, comme nous l'indique la définition de care donnée par Berenice Fischer et Joan Tronto:

\begin{abstract}
"Au niveau plus général, nous suggérons que le care soit considéré comme une activité générique, qui comprend tout ce que nous faisons pour maintenir, perpétuer et réparer notre monde, de sorte que nous puissions y vivre aussi bien que possible. Ce monde comprend nos corps, nous-mêmes et notre environnement, tous éléments que nous cherchons à relier en un réseau complexe, en soutien à la vie" (Fischer et Tronto, 1990: 40).
\end{abstract}

Le care, on le voit, se définit précisément en tant qu'actions pour "maintenir, perpétuer et réparer notre monde" et pour la sauvegarde de la vie. Gilligan souligne également que, dans les réponses au dilemme de Heinz, celle d'Amy, fillette de 11 ans au moment des entretiens analysés dans In A Different Voice (Gilligan, 1982), va dans cette même direction: "elle ne s'intéresse pas aux actions en tant que telles, abstraitement, mais à leur contexte et à la préservation de la vie" (Nurock, 2010: 63).

Par conséquent, les héroïnes des romans analysés agissant pour le maintien de la vie proposent un autre exemple d'héroïsme: un héroïsme de genre, qui se caractérise en premier lieu, non par un élan vers la mort, mais par un engagement pour la sauvegarde de la vie.

La première caractéristique de cet héroïsme de genre dont les travailleuses de care protagonistes des romans analysés nous fournissent des exemples, est la priorité du maintien de la vie.

Ensuite, le care se base sur l'existence d'une relation. Plus précisément le travail et l'éthique de care se situent exclusivement dans un contexte relationnel. Certes, les relations de care sont souvent des relations de 
dépendance, asymétriques, injustes, mais en dépit de tout cela, elles font partie de la vie de chacune, même si "idéalement, les relations de dépendance sont destinées à être surmontées car elles sont perçues comme des menaces pesant sur l'autonomie des individus" (Garrau et Le Goff, 2010: 15).

Au contraire I'héroïsme, comme catégorie neutre masculine, conçu dans et par le système patriarcal, se base sur des personnages qui ne sont pas simplement autonomes, ils sont l'exaltation même d'une autosuffisance absolue: le héros n'a besoin de personne! Selon Bilat et Haver, cette forme exacerbée d'autonomie est même l'une des caractéristiques qui définit un héros"il doit être auto-suffisant en termes de quête à accomplir, c'est-à-dire qu'il agit pour des motifs moraux supérieurs (on finit par croire) en mettant en second plan les aspects relationnels (amoureux, amicaux) de son existence" (Bilat et Haver, 2011: 18).

Jean Marie Apostolidès écrit que: "La culture héroïque se présente comme une célébration de la masculinité. Non seulement les femmes y tiennent une moindre place, mais on peut dire de cette culture qu'elle se construit à partir de la répression du pôle féminin, ou du moins, de sa subordination." (Apostolidès, 2011: 46).

Paola Bono et Bia Sarasini écrivent:

“D'une certaine façon le discours critique s'est arrêté à une épique qui chante 'les armes et I'homme' ou aussi: 'les femmes, les chevaliers, les armes et les amours', ou encore le cœur de l'aventure héroïque sont toujours les hommes, tandis que la présence des femmes est inévitablement reliée à un récit d'amour romantique" (Bono et Sarasini, 2014: 119).

Les deux auteures italiennes soulignent ici comment la critique littéraire oppose une résistance à dégager la définition d'exploit héroïque à des catégories qui ont été établies dans le passé et qui sont de genre universel masculin.

Donc, l'héroïsme fait partie de ces catégories neutres, universelles, en réalité masculines.

Bilat et Haver se disent eux aussi conscients des risques qu'il y a à parler $d^{\prime \prime \prime}$ "héroïsme". En effet l'archétype auquel cette catégorie fait référence ne devrait pas être genré, mais, de fait, il prend forme dans les personnages masculins de fiction. Cependant, ils décident d'utiliser le schéma de l'héroïsme faussement neutre pour chercher ce qu'est un 
"héros féminin". Le résultat auquel ils parviennent est la fabrication du portrait de plusieurs "héros féminins", c'est-à-dire d'héroïnes telles que le patriarcat les a façonnées: "There has been a persistent aura of inscrutability and discomfort surrounding feminine archetypes instead they are prescriptions for the gender system rather than revelations of the archetypal feminine (...) These revisions have been accepted as cultural fiat" (Powers, 1991: 6).

Comme le dénonce Meredith Powers, si on cherche les caractéristiques des héroïnes faisant référence à un héroïsme masculin, on ne pourra trouver que des exemples de personnages femmes telles que l'universel masculin les imagine.

Par exemple, les héros féminins analysés peuvent être physiquement puissants et violents autant et plus que les héros masculins, mais elles "doivent ajouter une certaine beauté du geste et conserver un corps désirable afin de répondre aux critères de la féminité dont elles ont de la peine à se déprendre" (Bilat et Haver, 2011: 26). Lara Croft, héros féminin du jeu-vidéo Tomb Raider, contrairement à ces homologues hommes pour lesquels les jeux prévoient seulement des positions d'attaque, peut être mise à quatre pattes par les joueurs! SheHulk, au contraire de Hulk, réussit à parler même quand elle devient verte (Hulk fait seulement des cris très gutturaux) et son corps reste très sexy, le gigantisme qu'il subit se situant surtout au niveau des seins!

On voit, alors, que même la catégorie d'"héroïsme", comme celle $d^{\prime \prime \prime}$ "héroïsation", nécessitent un usage critique, parce que leur sens, dans notre société, comporte des risques pour les femmes. Ce danger a été reconnu par lbos, comme on le verra plus loin, quand elle écrit que I'héroïsation des nounous est un énorme risque politique, et aussi par Arlie Hochschild (1990).

Au moment où la sociologue nord-américaine (Hochschild, 1990: 24) décrit la femme de la couverture du Time, souriante, soignée, avec les cheveux longs, tenant sa petite fille par la main, elle souligne les risques de cette héroïsation, ceux qui viennent de ce qu'ainsi représenté: I'héroïsme des femmes fortifie une division sexuelle du travail qui prévoit que les hommes ne s'occupent pas de l'entretien de la maison et de la famille. 
Lilian Robinson voit également un risque de discrimination de genre et de classe dans la création d'un "héros féminin", typique de notre société libérale contemporaine; elle définit comme "superwoman":

\begin{abstract}
"Someone who is competent professional and also a competent homemaker and mother. But this superwoman label bespeaks an underlying story about class as well as gender, because that heroic epithet is rarely applied to a woman raising a houseful of kids and her earnings as waitress or a switchboard operator or a domestic servant" (Robinson, 2004: 22).
\end{abstract}

À ce propos, dès 1979 Michele Wallace avait souligné comment I'héroïsation peut être aussi une histoire de race, la femme étant vue ainsi: "she is less femininely helpless but also really more of a woman in that she is the embodiment of Mother earth... In other words, she is a superwoman" (Wallace, 1979: 107).

Les dangers d'une représentation héroïque des femmes, qui adhère au schéma de l'héroïsme neutre masculin, touchent aussi les personnages des ménagères. Dans un système de représentation de l'exploit héroïque comme celui qui est dominant dans la société patriarcale, le risque est que "derrière toute housewife se cache une femme létale" (Bilat et Haver, 2011: 60). Les exemples sont très nombreux, ils vont de la série télévisée Desperates Housewives dont le créateur a inventé aussi Devious Maids, qui a récemment commencé à être diffusée en France, jusqu'au film Mr et Mrs Smith dans lequel Angelina Jolie cache un arsenal dans le four.

Dans tous ces cas, à côté de la force brutale, de la violence, dont font preuve ces femmes au foyer, on peut noter une hyper-sexualisation. Par exemple quand dans le film Au revoir à jamais Charly Baltimore, interprétée par Geena Davis, celle-ci décide d'adopter son ancienne identité d'agent du FBI, elle se débarrasse de ses vêtements chastes de mère de famille dévouée et endosse une robe en cuir. Le résultat est que "Charly est autant un monstre, cherchant à condenser toutes sortes d'identités, féminines ou non -qu'une forme versatile du héros féminin-" (Bilat et Haver, 2011: 63).

On peut ainsi observer qu'une héroïne créée à partir des attributs de I'héroïsme faussement neutre, est un monstre, car son personnage doit avoir à la fois les caractéristiques d'un héros, c'est-à-dire la force physique et le recours à la violence, mais au même temps elle doit 
répondre aux stéréotypes féminins, avoir un corps et des vêtements sexy.

La philosophe féministe italienne Annarosa Buttarelli écrit à ce propos:

"Il ne faut pas hésiter à créer ce vide, à arracher de la tradition littéraire masculine des personnages femmes jamais honorées par des compilateurs de généalogies culturelles et que ce vide soit justement le trou laissé par ces déchirures. Se rallier à la tradition patriarcale, même dans une perspective apparemment enrichissante, pour la critiquer et y réinsérer les femmes, engendre une monstruosité: une femme avec le pied dans différents souliers, donc souvent à terre, car elle est tombée; ou une femme avec deux têtes et un cœur" (Buttarelli 2002: 151).

Pour cette raison, Buttarelli incite à arracher les personnages femmes de la tradition patriarcale sans avoir peur des vides qui se forment, ainsi, à leur place.

Par conséquent, si on s'écarte des héros féminins, monstres créés par des généalogies culturelles masculines, on se trouvera face à un espace vide, un espace blanc pour s'interroger sur ce qu'est une héroïne dans le contexte d'un héroïsme de genre.

\section{Ce oue fait l'Ethnographie (QUAND elle est morale et POLITIQUE)}

Dans Qui gardera nos enfants?, analyse ethnographique du travail des nounous de la Côte d'Ivoire travaillant en région parisienne, Caroline lbos aborde la question de I'héroïsation des travailleuses qu'elle voit comme un risque politique énorme:

"Particulièrement courageuse dans un monde où les femmes en général sont courageuses, la nounou est le courage en personne. Mais cette identification la prend au piège du solipsisme des pauvres solitaires: finalement, il lui reste trop peu de temps, et peut-être plus aucune force, pour contester l'injustice de sa situation et s'engager dans de légitimes luttes collectives (...) En quelque sorte, l'énergie dépensée pour mettre en œuvre son œuvre de courage écrase politiquement la nounou" (Ibos, 2012: 196). 
Cependant dans l'épilogue de son texte, lbos se réfère aux nounous qu'elle a interviewées, précisément avec le terme "héroïnes": "dans ce livre, j'ai laissé une large place à la description et à la parole des héroïnes, tant il me semblait que, de cette description (...) une force critique émergeait" (Ibos, 2012: 224). La sociologue française explique qu'elle a choisi de fonder sa recherche justement sur les témoignages de ces femmes.

Toutefois, le fait qu'lbos souligne le risque politique d'héroïsation des nounous et en même temps déclare avoir basé son ouvrage sur les mots des héroïnes, protagonistes des histoires racontées, n'est pas une contradiction.

Le choix de baser la recherche sur les histoires des héroïnes, des nounous protagonistes, répond au constat que dans la relation de domination de l'employeuse sur la nounou, il y a une inversion du rapport traditionnel entre savoir et pouvoir: les employeuses ignorent les histoires de leurs nounous. Dans la plupart des cas elles ne savent pas d'où les nounous arrivent le matin quand elles entrent dans leurs maisons, ni où elles vont le soir quand elles ont terminé leur travail qui consiste à s'occuper de leurs enfants. Le manque de connaissance de ces histoires de vie est également à la base de l'absence de reconnaissance de leurs droits sociaux: "I'employeuse méprise la nounou (...) parce que la relation entre les deux femmes s'effectue au mépris de l'histoire singulière de la seconde" (Ibos, 2012: 180). Ibos décide de recueillir des récits de vie des héroïnes travailleuses domestiques, geste qui peut se lire comme une réponse au mépris des employeuses qui ignorent leurs histoires. Ainsi, consacrer des pages, un texte, aux récits de vie des nounous devient un acte politique.

Mais le livre ne se limite pas au recueil de ces récits. Il tente de saisir comment opère l'invisibilisation, par leurs employeuses, des personnes qui font le ménage et s'occupent des enfants. Il donne une consistance aux points de vue des employeuses qui relatent les tensions avec les employées. Les tensions, qui éclatent rarement en conflit ouvert, portent davantage sur le ménage, l'ordre tant matériel que symbolique de l'appartement bourgeois, que sur les soins aux enfants. L'étrangère qui entre dans la maison doit respecter un ordre qui n'est pas facile à discerner ou à décrypter comme le disent aussi bien les récits des employeuses que ceux des employées. La réticence de ces dernières par rapport au rangement est une résistance politique, ce sur quoi 
s'accordent également les employeuses quand elles parviennent à se mettre à la place de l'employée.

La relation est problématique puisqu'elle vient mettre "toute la misère du monde" au centre de leur appartement. Mais c'est pourtant à cette misère-là que ces mêmes employeuses doivent de pouvoir mener leur vie de professionnelles, et, pour celles qui vivent en couple, de pouvoir mener une vie de couple à l'abri des conflits domestiques sur l'ordre, le ménage, la propreté. La domination de classe qui se cristallise dans les questions d'ordre domestique peut aussi de comprendre selon Caroline lbos comme un déplacement de la domination masculine, l'emploi d'une travailleuse domestique étant une "variable d'ajustement" des arrangements entre les sexes.

La relation de subordination est rendue palpable dans sa complexité. Le mépris circule dans les récits faits par les employeuses qui, en dehors de l'état civil et de l'origine supposée de la personne qu'elle emploie, ignorent tout de la personne et de son histoire.

Pourtant cette ignorance ne peut être rabattue sur un mépris de classe car cet aveuglement à la personne qui soutient l'ordre domestique peut être le fait d'une employeuse qui, par ailleurs, est engagée dans des mobilisations dont celle des sans-papiers. Comment l'employeuse peutelle être en même temps engagée et indifférente, aveugle à la personne qui vient chaque jour chez elle?

L'indifférence à la vie de la personne employée est rendue possible par une dissociation: la personne employée est vue comme une sorte d'électron libre, elle est détachée des catégories ou des collectifs immigrées, femmes, pauvres- qui pourraient spécifier sa situation en référence à ces entités collectives. Ce détachement, cette dissociation est l'agencement qui dépolitise le quotidien, lequel finit par apparaître comme un registre séparé du social.

À la différence du récit littéraire centré sur une relation entre deux personnes même si cette relation est située dans un paysage social qui compose une part importante de l'intrigue, le compte rendu ethnographique de Caroline lbos est construit sur plusieurs histoires (22 employeuses, 13 nounous). Pluraliser ainsi le sujet de l'histoire -même s'il est fréquemment question dans le livre de "la" nounou comme une sorte d'idéal type qui vient synthétiser une position sociale à partir de plusieurs situations- est une manière, ou si l'on préfère une méthode, 
de remettre "I'objet politique" dans le flux de la vie. Cet objet-là, qu'on l'appelle politique, collectif ou social, est mieux figuré, semble-t-il, par une multitude que par une relation privilégiée. Et la conflictualité de la relation replacée dans sa dimension structurelle et collective prend de ce fait une tonalité différente, sourde et violente à la fois.

\section{LA VRAIE VIE ET LA FICTION}

L'exemple de La Porte souligne la dichotomie entre la vraie vie et la fiction romanesque. Magda écrit ce roman pour réparer sa faute à l'égard d'Emerenc. La fiction permet la réparation. Et raconte le miracle de la résolution du conflit. Dans la vraie vie Emerenc est morte. Et Magda Szabò raconte cette trahison et son effet sur Emerenc qui se laisse mourir. Magda Szabò se sent-elle mieux d'avoir raconté sa trahison? Estce l'aveu qui est réparateur? Pour qui?

Les écrivain(e)s ont besoin de connaître les histoires des employées, et si Magda Szabò le fait pour réparer sa faute à l'égard d'Emerenc, d'autres font de cette curiosité et de l'intérêt pour l'histoire de leur employée, le ressort positif de l'écriture. Dans certains récits littéraires, la connaissance de l'histoire de vie de la travailleuse de care engendre un processus de reconnaissance de la vulnérabilité humaine et par conséquent du besoin du care. Par exemple, l'écrivaine italienne Lalla Romano questionne la nounou Maria (1953), personnage dont dérive le titre du roman éponyme, pour connaître et reconnaître la femme qui s'occupe de son fils, la seule personne qui selon elle est en mesure de le faire. Dans Slow Man de J.M. Coetzee (2005), le personnage de l'écrivaine Elisabeth Costello semble entrer dans l'histoire justement pour montrer à Paul Rayment, le personnage du care-receiver qui a été amputé, et aux lectrices et lecteurs, son ignorance de la vie de l'aide-soignante Marijana, à travers des questionnements qui, à l'inverse, nous rendent visible l'histoire de la vie de cette travailleuse de care.

Lors de la publication du livre Qui gardera les enfants?, plusieurs des nounous protagonistes du livre de Caroline lbos ont été interviewées. Propulsées dans l'espace public médiatique pour un bref moment, elles ont profité de cet élan pour s'organiser et constituer un syndicat.

L'effet qu'a pu avoir le livre sur les employeuses n'a pas laissé de trace, même si l'on peut se demander si certains articles de presse sur le livre 
parus au moment de sa sortie n'expriment pas le point de vue des employeuses-eurs par leurs interrogations et remarques naïves.

Comme l'ont montré un certain nombre d'études - notamment le travail de Pascale Molinier (2013)- I'autonomisation de certaines femmes, en France et ailleurs, par le travail, et parallèlement par le développement des systèmes de garde d'enfants, des services à domicile, etc., s'est aussi faite non par un transfert des tâches aux hommes, ou une organisation plus juste du travail du care, mais par la mise d'autres femmes au service de ces femmes et de leur "autonomisation". Il ne s'agit évidemment pas d'ironiser sur ces femmes, devenues employeuses: c'est toujours à elles de porter la charge morale, et administrative, de l'emploi à domicile, de prendre la place de contremaîtres permettant ainsi à certains de s'exempter en outre de ce rapport d'employeur à employée. Il s'agit bien plutôt, comme souvent dans le care, de faire voir ce qui est juste sous notre nez: que les tâches de care, traditionnellement dévolues aux femmes (au foyer ou non) doivent toujours être accomplies même si certains -occidentaux, favorisés- en sont déchargés parce qu'ils peuvent payer pour cela. La division sociale et mondiale du travail de care rappelle l'importance qu'il y a à (re)penser ensemble care et délégation du travail.

Comme l'écrit Sandra Laugier:

"Le care, en suggérant une attention nouvelle à des détails inexplorés de la vie ou à des éléments qui sont négligés, nous confronte à nos propres incapacités et inattentions, mais aussi et surtout à la façon dont elles se traduisent ensuite en théorie. L'enjeu des éthiques du care s'avère épistémologique en devenant politique: elles veulent mettre en évidence le lien entre notre manque d'attention à des réalités négligées et le manque de théorisation (ou, de façon plus directe, le rejet de la théorisation) de ces réalités sociales 'invisibilisées'" (Laugier, 2011: 58).

Une expression consacrée en sociologie que l'on doit à Marcel Mauss (2013) —“fait social total"- pourrait bien être détournée: le care est bien ce "travail social total" qui est aussi, et peut-être avant tout, un travail de l'attention, une façon différente de percevoir et de valoriser les activités humaines comme le montrent et le disent les récits de Szabò et Ibos. 


\section{BIBLIOGRAPHIE}

Apostolidès, J. M., 2011, Héroïsme et victimisation, Éditions du Cerf, Paris.

Bilat, L., Haver, G., (Eds.), 2011, Le héros était une femme, Éditions Antipodes, Lausanne.

Buttarelli, A., 2002, Tabula rasa, en Diotima, Approfittare dell'assenza, Liguori, Napoli, pp. 143-154.

Coetzee, J.M., 2005, Slow Man, Penguin Books, London.

Bono, P., Sarasini B., (Eds.), 2014, Epiche. Altre imprese, altre narrazioni, lacobelli Editori, Roma.

De Lauretis, T., 1982, Alice doesn't, Indiana University Press, Bloomington.

Dictionnaire Culturel en langue française, 2005, Dictionnaires Le Robert, Paris.

Fischer, B. M., Tronto J. C., 1990, "Toward a Feminist Theory of Care", en E. K. Abel y K. Nelson (Eds.), Circles of Care, State University of New York Press, Albany, pp. 36-54.

Garrau, M., Le Goff A., (Eds.), 2010, Care, justice et dépendance. Introduction aux théories du care, PUF, Paris.

Gilligan, C., 1982, In a Different Voice: Psychological Theory and Women's Development, Harvard University Press, Harvard.

Grand Larousse de la langue française, 1989, Larousse, Paris.

Hirata, H., avec Labari, B., Falquet, J., Le Feuvre, N., Kergoat, D., Sow, F., (Eds.), 2010, Le sexe de la mondialisation. Genre, classe, race et nouvelle division du travail, Presses de Sciences Po, coll. Académique, Paris.

Hochschild, A., with Machung, A., 1990, The second shift, Avon Books, New York.

Ibos, C., 2012, Qui gardera nos enfants?, Flammarion, Paris.

Laugier, S., 2011, "Le care, le souci du détail et la vulnérabilité du réel", en Raisons publiques, $\mathrm{n}^{\circ} 14$, Avril 2011, pp. 39-58.

Le Grand Larousse Encyclopédique, 1962, Librairie Larousse, Paris.

Le Nouveau Petit Robert. Dictionnaire de la langue française, 2008, Dictionnaires Robert, Paris.

Lions, D., 1987, Gender and immortality, Princeton University Press, Princeton.

Mauss, M., 2013 [1923], Sociologie et anthropologie, PUF, Paris. 
Molinier, P., 2013, Le travail du care, La Dispute, Paris.

Nurock, V., (Eds.), 2010, Carol Gilligan et l'éthique du care, PUF, Paris.

Powers, M. A., 1991, The heroine in western literature, Mac Pharland, London.

Robinson, L., 2004, Wonder woman. Feminism and superheroes, Routledge, New York.

Romano, L., 1953, Maria, Einaudi, Torino.

Szabò, M., 2003, La porte, Viviane Hamy, Paris.

Tronto, J., 2009, Un monde vulnérable. Pour une politique du care, La Découverte, Paris.

Trésor de la langue française. Dictionnaire de la langue du XIXème et du XXème siècle, 1981, Centre National de la recherche scientifique, Paris.

Wallace, M., 1979, Black Macho and the myth of superwoman, Dial Press New York. 